\title{
The light asymptotic limit of conformal blocks in Toda field theory
}

\author{
Hasmik Poghosyan, ${ }^{a}$ Rubik Poghossian ${ }^{a}$ and Gor Sarkissian ${ }^{a, b}$ \\ ${ }^{a}$ Yerevan Physics Institute, \\ Alikhanian Br. 2, 0036 Yerevan, Armenia \\ ${ }^{b}$ Department of Physics, Yerevan State University, \\ Alex Manoogian 1, 0025 Yerevan, Armenia \\ E-mail: hasmikpoghos@gmail.com, poghos@yerphi.am, \\ gor.sarkissian@ysu.am
}

ABSTRACT: We compute the light asymptotic limit of $A_{n-1}$ Toda conformal blocks by using the AGT correspondence. We show that for certain class of CFT blocks the corresponding Nekrasov partition functions in this limit are simplified drastically being represented as a sum of a restricted class of Young diagrams. In the particular case of $A_{2}$ Toda we also compute the corresponding conformal blocks using conventional CFT techniques finding a perfect agreement with the results obtained from the Nekrasov partition functions.

KEYWORDS: Conformal and W Symmetry, Supersymmetric gauge theory

ArXiv EPrint: 1602.04829 


\section{Contents}

1 Introduction 1

2 The light asymptotic limit of the Nekrasov partition functions 3

2.1 The Nekrasov partition functions of $\mathcal{N}=2$ SYM theory 3

2.2 Preliminaries on $A_{n-1}$ Toda CFT and AGT relation 4

2.3 Light asymptotic limit 6

2.4 Nekrasov partition function of $\mathcal{N}=2 \mathrm{SYM}$ theory in the light asymptotic limit 8

3 Light asymptotic limit for the four point block in $W_{3} \quad 11$

4 Discussion $\quad 14$

$\begin{array}{ll}\text { A The integral calculation } & 14\end{array}$

$\begin{array}{ll}\text { B The second order terms for } W_{3} & 16\end{array}$

\section{Introduction}

Semiclassical limits play important role since they link quantum physics to the Lagrangian approach. In the Liouville and Toda field theories there are three semiclassical limits: mini-superspace [1-4], the light and heavy [4-6]. All three asymptotics are the large central charge limits. The difference comes in the treatment of the primary fields. In the minisuperspace limit one considers a limit where only the zero mode dynamics survives. In this limit the Liouville and Toda field theories reduce to the corresponding quantum mechanical problems $[1,2,4]$. In the light asymptotic limit one keeps the conformal dimensions fixed. Then the correlation functions are given by the finite dimensional path integral over solutions of the equations of motion with a vanishing energy-momentum tensor. And finally in the heavy asymptotic limit the conformal dimensions blow up, scaling as the classical action and correlation functions are given by the exponential of the action evaluated over the singular solutions.

To be more specific recall that primary fields in the Liouville and Toda field theories are related to the vertex operators $V_{\alpha}=e^{i \alpha \phi}$. The spectrum is given by $\alpha=\frac{Q}{2}+i P$. In the light asymptotic limit we set $\alpha=\eta_{l} b$ and keep $\eta_{l}$ fixed for $b \rightarrow 0$, whereas in the heavy asymptotic limit we take $\alpha=\frac{\eta_{h}}{b}$ and hold $\eta_{h}$ fixed again for $b \rightarrow 0$. In the minisuperpsace limit one should take for some of the vertex operators $\alpha=\eta_{m} b$ and for some $P=\eta_{m} b$.

These semiclassical limits were used in $[3,4,6]$ to relate the quantum three-point functions in the Liouville and Toda theories with the corresponding classical actions. In papers [7-9] these techniques were generalized to the boundary Liouville and Toda theories. 
The heavy and light asymptotic limits were reconsidered in [10] also for complex solutions of the analytically continued Liouville theory. In [11] both limits were used in the Liouville field theory with defects.

Discovery of AGT correspondence [12-15] relating 2d CFT conformal blocks to the Nekrasov partition function $[16,17]$ in $\mathcal{N}=2$ supersymmetric gauge theory provides powerful tools to investigate CFT correlators using gauge theory methods or alternativly to apply advanced CFT methods in gauge theory (see e.g. [18, 19]. The essential point here is the fact that there are explicit combinatorial formulas for the Nekrasov partition function [20, 21], which now can be successfully applied in 2d CFT.

The heavy asymptotic limit in the AGT context was considered in [22-24]. In [25-27] the light asymptotic limit was used to test the AGT correspondence.

Investigation of the light asymptotic limit of the Nekrasov functions for $\mathrm{U}(2) N=$ 2 gauge theory in $[25,27]$ revealed a very interesting fact, that in this limit only the Young diagrams consisting of a single row contribute. This could be anticipated since in the light asymptotic limit the infinite Virasoro symmetry reduces to SL(2) algebra whose representations are classified with one row Young tableaux.

The sum over these one-row Young tableaux yields the Gauss Hypergeometric function, which is the well known light asymptotic limit of the Virasoro conformal blocks [28].

In [26] the AGT correspondence was tested in the light asymptotic limit between conformal blocks of $A_{2}$ Toda field theory and Nekrasov functions of $\mathrm{U}(3) \mathrm{N}=2$ gauge theory. Namely some conformal blocks for $A_{2}$ Toda field theory were computed in this limit and the result was checked against the corresponding limit of the Nekrasov functions up to the 5-th order terms. But such an elegant picture as it was found for the Liouville field theory was missing.

In this paper we consider the light asymptotic limit of the $\mathrm{U}(n)$ Nekrasov partition functions for an arbitrary $n$. We find that for the certain choice of fields the Nekrasov partition functions in the light asymptotic limit are simplified drastically and given by the sum over Young diagrams having at most $n-1$ rows. We compute the corresponding $W_{3}$ conformal block using the light asymptotic integral representation and found perfect agreement with the two-row Nekrasov partition functions. Note that in the light asymptotic limit the $W_{n}$ symmetry reduces to $\operatorname{SL}(n)$ group [26, 29] and this already hints on the existence of the limiting procedure where survive only Young diagrams corresponding to the $\mathrm{SL}(n)$ representations.

The paper is organized as follows. In section 2 we compute the light asymptotic limit of the Nekrasov partition functions. In subsection 2.1 we review the necessary facts on the Nekrasov partition functions. In subsection 2.2 we review Toda conformal field theory and the AGT relation. In subsection 2.3 we explain the details on the light asymptotic limit and show that choosing the data as it is specified in eq. (2.18) and (2.19) truncates the Nekrasov functions in the light asymptotic limit to the sum over Young tableaux containing at most $n-1$ rows. In subsection 2.4 we compute the Nekrasov partition function in the light asymptotic limit. The formulas (2.34) and (2.35) are our main result. In section 3 we compute the corresponding conformal block in $A_{2}$ Toda field theory using that in the 


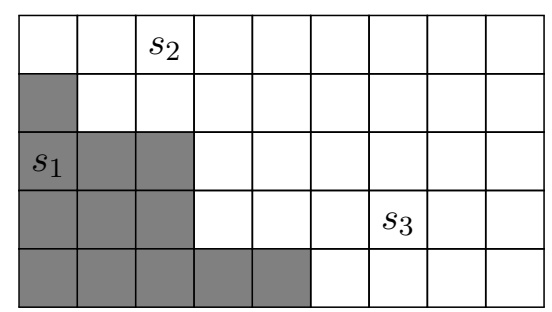

Figure 1. Arm and leg length with respect to a Young diagram (pictured in gray): $A\left(s_{1}\right)=1$, $L\left(s_{1}\right)=2, A\left(s_{2}\right)=-2, L\left(s_{2}\right)=-3, A\left(s_{3}\right)=-2, L\left(s_{3}\right)=-4$.

light asymptotic limit conformal blocks admit an integral representations. In appendix A the details of the integral calculation in section 3 are delivered.

\section{The light asymptotic limit of the Nekrasov partition functions}

\subsection{The Nekrasov partition functions of $\mathcal{N}=2$ SYM theory}

Consider $\mathcal{N}=2$ SYM theory with gauge group $\mathrm{U}(n)$ and $2 n$ fundamental (more precisely $n$ fundamental plus $n$ anti-fundamental) hypermultiplets in $\Omega$-background. The instanton part of the partition of this theory can be represented as

$$
Z_{\text {inst }}=\sum_{\vec{Y}} F_{\vec{Y}} z^{|\vec{Y}|}
$$

where $\vec{Y}$ is an array of $n$ Young diagrams, $|\vec{Y}|$ is the total number of boxes and $z$ is the instanton counting parameter related to the gauge coupling in a standard manner. The coefficients $F_{\vec{Y}}$ are given by

$$
F_{\vec{Y}}=\prod_{u=1}^{n} \prod_{v=1}^{n} \frac{Z_{b f}\left(a_{u}^{(0)}, \varnothing \mid a_{v}^{(1)}, Y_{v}\right) Z_{b f}\left(a_{u}^{(1)}, Y_{u} \mid a_{v}^{(2)}, \varnothing\right)}{Z_{b f}\left(a_{u}^{(1)}, Y_{u} \mid a_{v}^{(1)}, Y_{v}\right)}
$$

where

$$
\begin{aligned}
& Z_{b f}(a, \lambda \mid b, \mu)= \\
& =\prod_{s \in \lambda}\left(a-b-\epsilon_{1} L_{\mu}(s)+\epsilon_{2}\left(1+A_{\lambda}(s)\right)\right) \prod_{s \in \mu}\left(a-b+\epsilon_{1}\left(1+L_{\lambda}(s)\right)-\epsilon_{2} A_{\mu}(s)\right) .
\end{aligned}
$$

Here $A_{\lambda}(s)$ and $L_{\lambda}(s)$ are correspondingly the arm-length and leg-length of the square $s$ towards the Young tableau $\lambda$, defined as oriented vertical and horizontal distances of the square $s$ to outer boundary of the Young tableau $\lambda$ (see figure 1).

Let us clarify our conventions on gauge theory parameters $a_{u}^{(0,1,2)}, u=1,2, \ldots, n$. The parameters $a_{u}^{(1)}$ are expectation values of the scalar field in vector multiplet. Without loss of generality we'll assume that the "center of mass" of these expectation values is zero

$$
\bar{a}^{(1)}=\frac{1}{n} \sum_{u=1}^{n} a_{u}^{(1)}=0 .
$$


In fact this is not a loss of generality since a nonzero center of mass can be absorbed by shifting hypermultiplet masses. Furthermore $a_{u}^{(0)}\left(a_{u}^{(2)}\right)$ are the masses of fundamental (anti-fundamental) hypers. Finally the $\epsilon_{1}, \epsilon_{2}$ are the $\Omega$-background parameters. Sometimes we will use the notation $\epsilon=\epsilon_{1}+\epsilon_{2}$.

Due to AGT duality, this partition function is directly related to specific four point conformal block in $2 \mathrm{~d} A_{n-1}$ Toda field theory. Before describing this relation let us briefly recall few facts about Toda theory.

\subsection{Preliminaries on $A_{n-1}$ Toda CFT and AGT relation}

These are 2d CFT theories which besides the spin 2 holomorphic energy momentum $W^{(2)}(z) \equiv T(z)$ are endowed with additional higher spin $s=3,4, \ldots, n$ currents $W^{(3)}$, $\ldots, W^{(n)}$ with Virasoro central charge conventionally parameterised as

$$
c=n-1+12 Q^{2},
$$

where the vector "background charge"

$$
Q=\rho(b+1 / b)
$$

with $\rho$ being the Weyl vector of the algebra $A_{n-1}$ and $b$ is the dimensionless coupling constant of Toda theory. In what follows it would be convenient to represent the roots, weights and Cartan elements of $A_{n-1}$ as $n$-component vectors with the usual Kronecker scalar product, subject to the condition that sum of components is zero. Of course this is equivalent to more conventional representation of these quantities as diagonal traceless $n \times n$ matrices with the pairing given by trace. In this representation the Weyl vector is given by

$$
\rho=\left(\frac{n-1}{2}, \frac{n-3}{2}, \ldots, \frac{1-n}{2}\right) \quad \text { or } \quad \rho_{u}=\frac{n+1}{2}-u
$$

and for the central charge we'll get

$$
c=(n-1)\left(1+n(n+1) q^{2}\right),
$$

where for the later use we have introduced the parameter

$$
q=b+\frac{1}{b} .
$$

For further reference let us quote here explicit expressions for the highest weight $\omega_{1}$ of the first fundamental representation and for its complete set of weights $h_{1}, \ldots, h_{n}\left(h_{1}=\omega_{1}\right)$

$$
\begin{aligned}
\left(\omega_{1}\right)_{k} & =\delta_{1, k}-1 / n ; \\
\left(h_{l}\right)_{k} & =\delta_{l, k}-1 / n .
\end{aligned}
$$

The primary fields $V_{\alpha}$ (here we concentrate only on left moving holomorphic parts) are parameterized by vectors $\alpha$ with vanishing center of mass. Their conformal weights are given by

$$
h_{\alpha}=\frac{\alpha(2 Q-\alpha)}{2} \text {. }
$$



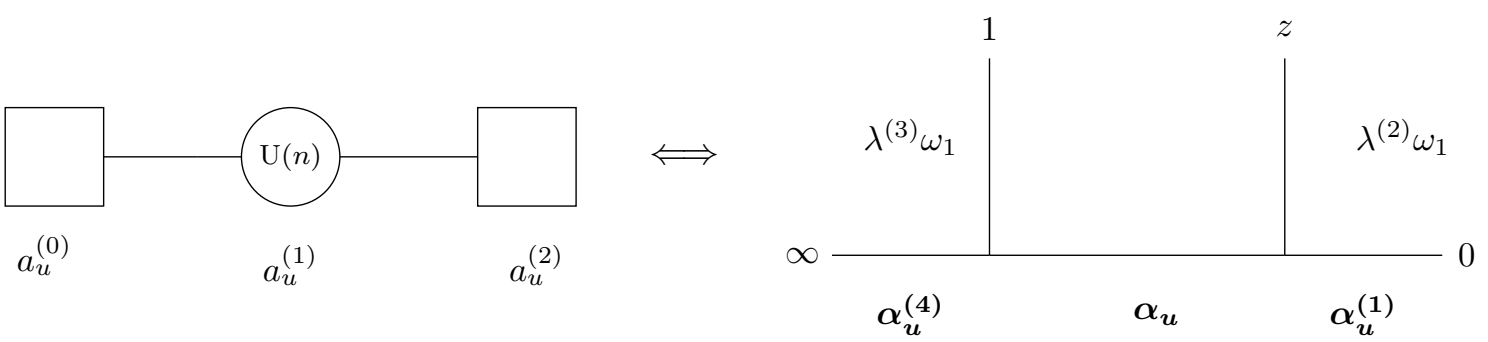

Figure 2. On the left: the quiver diagram for the conformal $\mathrm{U}(n)$ gauge theory. On the right: the diagram of the conformal block for the dual Toda field theory.

In what follows a special role is played by the fields $V_{\lambda \omega_{1}}$ with the dimensions:

$$
h_{\lambda \omega_{1}}=\frac{\lambda(n-1)}{2}\left(q-\frac{\lambda}{n}\right) \text {. }
$$

A four point block:

$$
\left\langle V_{\alpha^{(4)}}(\infty) V_{\lambda^{(3)} \omega_{1}}(1) V_{\lambda^{(2)} \omega_{1}}(z) V_{\alpha^{(1)}}(0)\right\rangle_{\alpha}=z^{h_{\alpha}-h_{\alpha^{(1)}}-h_{\lambda^{(2)} \omega_{1}} \mathcal{F}_{\alpha}}\left[\begin{array}{cc}
\lambda^{(3)} \omega_{1} \lambda^{(2)} \omega_{1} \\
\alpha^{(4)} & \alpha^{(1)}
\end{array}\right](z),
$$

where $\alpha$ specifies the $\mathrm{W}$-family running in s-channel, is closely related to the gauge partition function $Z_{\text {inst }}$ see (2.15) (AGT relation). First of all, the instanton counting parameter $z$ gets identified with the cross ratio of insertion points in CFT block as it was already anticipated in (2.9) and the Toda parameter $b$ is related to $\Omega$-background parameters via

$$
b=\sqrt{\frac{\epsilon_{1}}{\epsilon_{2}}} .
$$

The map between the gauge parameters in (2.1) and conformal block parameters in (2.9) should be established from the following rules (see figure 2). To formulate them we define the rescaled gauge parameters

$$
A_{u}^{(0)}=\frac{a_{u}^{(0)}}{\sqrt{\epsilon_{1} \epsilon_{2}}} ; \quad A_{u}^{(1)}=\frac{a_{u}^{(1)}}{\sqrt{\epsilon_{1} \epsilon_{2}}} ; \quad A_{u}^{(2)}=\frac{a_{u}^{(2)}}{\sqrt{\epsilon_{1} \epsilon_{2}}} .
$$

- The differences between the "centers of mases" of the successive rescaled gauge parameters (2.11) give the charges of the "vertical" entries of the conformal block:

$$
\bar{A}^{(1)}-\bar{A}^{(0)}=\frac{\lambda^{(3)}}{n} ; \quad \bar{A}^{(2)}-\bar{A}^{(1)}=\frac{\lambda^{(2)}}{n} .
$$

- The rescaled gauge parameters with the subtracted centers of masses give the momenta of the "horizontal" entries of the conformal block:

$$
\begin{aligned}
& A_{u}^{(0)}-\bar{A}^{(0)}=Q_{u}-\alpha_{u}^{(4)} ; \\
& A_{u}^{(1)}-\bar{A}^{(1)}=Q_{u}-\alpha_{u} ; \\
& A_{u}^{(2)}-\bar{A}^{(2)}=Q_{u}-\alpha_{u}^{(1)} .
\end{aligned}
$$


Using (2.4), (2.5) and (2.11)-(2.13) we obtain the relation between the gauge and conformal parameters:

$$
\begin{aligned}
& \frac{a_{u}^{(0)}}{\sqrt{\epsilon_{1} \epsilon_{2}}}=-\alpha_{u}^{(4)}-\frac{\lambda^{(3)}}{n}+q\left(\frac{n+1}{2}-u\right) ; \\
& \frac{a_{u}^{(1)}}{\sqrt{\epsilon_{1} \epsilon_{2}}}=-\alpha_{u}+q\left(\frac{n+1}{2}-u\right) ; \\
& \frac{a_{u}^{(2)}}{\sqrt{\epsilon_{1} \epsilon_{2}}}=-\alpha_{u}^{(1)}+\frac{\lambda^{(2)}}{n}+q\left(\frac{n+1}{2}-u\right) .
\end{aligned}
$$

With all these preparations one can write the AGT correspondence between the Nekrasov function defined in (2.1) and the conformal block in (2.9) (see [13, 15]):

$$
Z_{\text {inst }}=(1-z)^{\lambda^{(3)}\left(q-\frac{\lambda^{(2)}}{n}\right)} \mathcal{F}_{\alpha}\left[\begin{array}{cc}
\lambda^{(3)} \omega_{1} & \lambda^{(2)} \omega_{1} \\
\alpha^{(4)} & \alpha^{(1)}
\end{array}\right](z) .
$$

\subsection{Light asymptotic limit}

In this paper we are interested in so called "light" asymptotic limit i.e. the central charge is sent to infinity (i.e. $b \rightarrow 0$ ) while keeping the dimensions finite. It follows from (2.7) that to reach this limit one can simply put

$$
\alpha_{u}^{(1)}=b \eta_{u}^{(1)} ; \quad \alpha_{u}^{(4)}=b \eta_{u}^{(4)} ; \quad \alpha_{u}=b \eta_{u}
$$

keeping all the parameters $\eta$ finite. As for the parameters $\lambda$ of the special fields $V_{\lambda \omega_{1}}$, there are two inequivalent alternatives:

(i) $\lambda=b \eta$ or

(ii) $n q-\lambda=b \eta$.

Though in both cases the conformal dimension takes the same value (see eq. (2.8))

$$
h=\frac{\eta(n-1)}{2}
$$

these fields are not identical, which can be seen e.g. from the fact that the zero mode eigenvalues of odd $\mathrm{W}$-currents for these fields have the same absolute values but opposite signs. In fact the fields $V_{b \eta \omega_{1}}$ and $V_{(n q-b \eta) \omega_{1}}$ can be considered as conjugate to each other in the usual sense, since their two point function is non-zero. It is easy to check that $V_{(n q-b \eta) \omega_{1}}$ is equivalent to $V_{b \eta \omega_{n-1}}\left(\omega_{n-1}\right.$ is the highest weight of the anti-fundamental representation) since the corresponding momentum parameters $Q-b \eta \omega_{1}$ and $Q-(n q-b \eta) \omega_{n-1}$ are related by a Weyl reflection.

In this paper we will investigate in great detail the case when $V_{\lambda^{(3)} \omega_{1}}$ is a light field of type (i) while $V_{\lambda^{(2)} \omega_{1}}$ is of type (ii). In other words we set

$$
\lambda^{(3)}=b \eta^{(3)} ; \quad n q-\lambda^{(2)}=b \eta^{(2)} .
$$




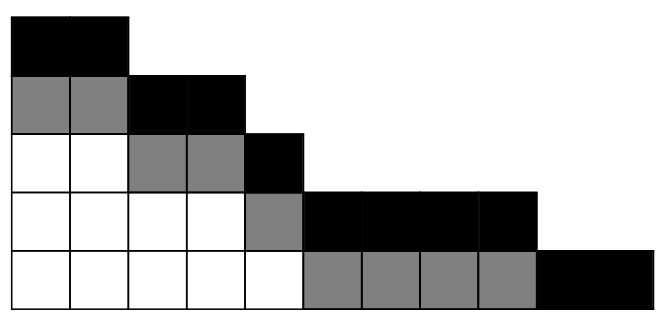

Figure 3. This picture shows that there are $Y_{v, 1}$ boxes such that $A_{Y_{v}}=0$ (painted black) and $Y_{v, 2}$ boxes with $A_{Y_{v}}=1$ (painted grey).

For such choice we will see below, that the corresponding instanton sum simplifies drastically and leads to a simple explicit expression for the conformal block. Note that this choice is very convenient since the prefactor in front of conformal block in (2.15) now goes to 1 in the light asymptotic limit. The opposite case when two special fields are of the same type, has been investigated in [26] in particular case of $A_{2}$ Toda. In the case considered in [26] the above mentioned prefactor survives.

Coming back to our case of interest using (2.18), (2.16) we can rewrite the AGT map (2.14) as

$$
\begin{aligned}
& a_{u}^{(0)}=-\epsilon_{1}\left(\eta_{u}^{(4)}+\frac{\eta^{(3)}}{n}\right)+\epsilon\left(\frac{n+1}{2}-u\right) ; \\
& a_{u}^{(1)}=-\epsilon_{1} \eta_{u}+\epsilon\left(\frac{n+1}{2}-u\right) ; \\
& a_{u}^{(2)}=-\epsilon_{1}\left(\eta_{u}^{(1)}+\frac{\eta^{(2)}}{n}\right)+\epsilon\left(\frac{n+3}{2}-u\right) .
\end{aligned}
$$

In view of (2.10) the small $b$ limit is equivalent to $\epsilon_{1} \rightarrow 0$. Hence we are interested in the $\epsilon_{1} \rightarrow 0$ limit of (2.2). We will see that the degree of $\epsilon_{1}$ (denote it by $N$ ) is non-negative for arbitrary array of Young diagrams $Y_{v}$ and that the degree $N=0$ (hence a finite non-zero limit exists) if and only if each Young diagram $Y_{v}(v=1,2, \ldots, n)$ has at most $v-1$ rows. From (2.2) we see that

$$
N=n_{1}+n_{2}-n_{3}
$$

with $n_{1}, n_{2}$ being the $\epsilon_{1}$ degrees of the first and second factors in the numerator of (2.2) respectively and $n_{3}$ is the $\epsilon_{1}$ degree of its denominator.

Let us derive $n_{1}$, using (2.3) for $Z_{b f}\left(a_{u}^{(0)}, \varnothing \mid a_{v}^{(1)}, Y_{v}\right)$ and inserting (2.19) we'll get

$$
\begin{aligned}
& Z_{b f}\left(a_{u}^{(0)}, \varnothing \mid a_{v}^{(1)}, Y_{v}\right)= \\
& =\prod_{s \in Y_{v}}\left(\epsilon_{1}\left(1+L_{\varnothing}(s)+\eta_{v}-\eta_{u}^{(4)}-\frac{\eta^{(3)}}{n}\right)+\epsilon(v-u)-\epsilon_{2} A_{Y_{v}}(s)\right) .
\end{aligned}
$$

A factor in (2.21) contributes to the degree of $\epsilon_{1}$ if its part proportional to $\epsilon_{2}$ vanishes. Evidently this happens when $A_{Y_{v}}(s)=v-u$. Since the box $s \in Y_{v}, A_{Y_{v}}(s) \geq 0$, we see that when $v=1$ the only admissible value for $u$ is $u=1$. It is obvious from figure 3 that 
there are exactly $Y_{1,1}$ boxes in $Y_{1}$ for which the arm-length vanishes (here and below we denote by $Y_{v, i}$ the number of boxes in the $i$ 'th row of diagram $Y_{v}$ ). When $v=2$, there are two admissible values $u=1$ or $u=2$. As in the previous case the number of the boxes with zero arm-length (case $u=2$ ) is equal $Y_{2,1}$. Similarly, a simple inspection shows that the number of boxes with unit arm-lengths (case $u=2$ ) are equal to $Y_{2,2}$. This analysis can be easily continued for other values of $v$ with result summarized in the table below

\begin{tabular}{|l|c|c|c|c|c|}
\hline & $\mathrm{u}=1$ & $\mathrm{u}=2$ & $\mathrm{u}=3$ & $\ldots$ & $\mathrm{u}=\mathrm{n}$ \\
\hline $\mathrm{v}=1$ & $Y_{1,1}$ & & & & \\
\hline $\mathrm{v}=2$ & $Y_{2,2}$ & $Y_{2,1}$ & & & \\
\hline $\mathrm{v}=3$ & $Y_{3,3}$ & $Y_{3,2}$ & $Y_{3,1}$ & & \\
\hline$\ldots$ & & & & $\ldots$ & \\
\hline $\mathrm{v}=\mathrm{n}$ & $Y_{n, n}$ & $Y_{n, n-1}$ & $Y_{n, n-2}$ & $\ldots$ & $Y_{n, 1}$ \\
\hline
\end{tabular}

Obviously the degree $n_{1}$ is nothing but the sum of all entries of this table.

$$
n_{1}=\sum_{u=1}^{n} \sum_{k=1}^{u} Y_{u, k}
$$

With almost identical arguments it is possible to show that $n_{2}=n_{1}$. Finally, an analogous consideration for the degree $n_{3}$ gives

$$
n_{3}=\sum_{u=1}^{n} \sum_{k=1}^{u} Y_{u, k}+\sum_{u=1}^{n} \sum_{k=1}^{u-1} Y_{u, k} .
$$

Thus for the total degree $(2.20)$ we get

$$
N=\sum_{u=1}^{n} Y_{u, u}
$$

Each term here is non-negative and in order to get a vanishing total degree $N=0$, the array of Young diagrams should satisfy the conditions $Y_{1,1}=Y_{2,2}=\cdots Y_{n, n}=0$, which means that each Young diagram $Y_{u}$ consists of at most $u-1$ rows.

\subsection{Nekrasov partition function of $\mathcal{N}=2$ SYM theory in the light asymptotic limit}

Now our purpose is to derive $F_{\vec{Y}}$ explicitly in the light asymptotic limit. To do this let us study the first factor in the numerator of (2.2) which, according to (2.3) and (2.19), is given by

$$
\begin{aligned}
& Z_{b f}\left(a_{u}^{(0)}, \varnothing \mid a_{v}^{(1)}, Y_{v}\right)= \\
& =\prod_{s \in Y_{v}}\left(\epsilon_{1}\left(1+L_{\varnothing}(s)+\eta_{v}-\eta_{u}^{(4)}-\frac{\eta^{(3)}}{n}\right)+\epsilon(v-u)-\epsilon_{2} A_{Y_{v}}(s)\right) .
\end{aligned}
$$


Let $Y_{v}^{(1)}$ be the set of such boxes $s$ of the Young diagram $Y_{v}$ (with at most $v-1$ rows) that the coefficient of $\epsilon_{2}$ vanishes in the respective factor of (2.25)), i.e.

$$
v-u-A_{Y_{v}}(s)=0 .
$$

This can happen only when $i \equiv v-u \geq 0$. Thus for the part of (2.25) under discussion we get

$$
Z_{b f}\left(a_{u}^{(0)}, \varnothing \mid a_{v}^{(1)}, Y_{v}^{(1)}\right)=\prod_{s \in Y_{v}^{(1)}} \epsilon_{1}\left(1+L_{\varnothing}(s)+\eta_{v}-\eta_{u}^{(4)}-\frac{\eta^{(3)}}{n}+v-u\right) .
$$

We have already seen in previous section that there are exactly $Y_{v, i+1}$ boxes, satisfying (2.26). These boxes are distributed in $Y_{v}$ in such a way that there is a single box on $j$-th column (denote it by $s_{j}$ ) for each $j=1, \ldots, Y_{v, i+1}$ (see figure 3 ). Taking into account that $L_{\varnothing}\left(s_{j}\right)=-j$, we can rewrite $(2.27)$ as

$$
Z_{b f}\left(a_{u}^{(0)}, \varnothing \mid a_{v}^{(1)}, Y_{v}^{(1)}\right)=\prod_{j=1}^{Y_{v, i+1}} \epsilon_{1}\left(\eta_{v}-\eta_{v-i}^{(4)}-\frac{\eta^{(3)}}{n}+1-j+i\right) .
$$

Now let's look on the alternative case of the set $Y_{v}^{(2)}$ of those boxes which do not satisfy (2.26) so that in the related factors we can safely set $\epsilon_{1}=0$. Again from (2.21) we'll get

$$
Z_{b f}\left(a_{u}^{(0)}, \varnothing \mid a_{v}^{(1)}, Y_{v}^{(2)}\right)=\prod_{s \in Y_{v}^{(2)}} \epsilon_{2}\left(v-u-A_{Y_{v}}(s)\right) .
$$

Carefully examining the cases $v-u-A_{Y_{v}}(s)>0$ and $v-u-A_{Y_{v}}(s)<0$ separately we get

$$
\begin{aligned}
& \prod_{u=1}^{n} \prod_{v=1}^{n} Z_{b f}\left(a_{u}^{(0)}, \varnothing \mid a_{v}^{(1)}, Y_{v}^{(2)}\right)= \\
& =\prod_{v=2}^{n} \prod_{i=1}^{v-1}\left((-)^{n-v-1-i}(n-v-1-i) !(v-i) ! \epsilon_{2}^{(n-1)}\right)^{Y_{v, i}}
\end{aligned}
$$

Combining (2.28) with (2.30) we obtain

$$
\begin{aligned}
& \prod_{u=1}^{n} \prod_{v=1}^{n} Z_{b f}\left(a_{u}^{(0)}, \varnothing \mid a_{v}^{(1)}, Y_{v}\right)= \\
& =\prod_{v=2}^{n} \prod_{i=0}^{v-2} \prod_{j=1}^{Y_{v, i+1}} \epsilon_{1}\left(\eta_{v}-\eta_{v-i}^{(4)}-\frac{\eta^{(3)}}{n}+1-j+i\right) \times \\
& \quad \times \prod_{v=2}^{n} \prod_{i=1}^{v-1}\left((-)^{n-v-1-i}(n-v-1-i) !(v-i) ! \epsilon_{2}^{(n-1)}\right)^{Y_{v, i}}
\end{aligned}
$$

Similar arguments for the second factor in the denominator of (2.2) lead to the expression

$$
\prod_{u=1}^{n} \prod_{v=1}^{n} Z_{b f}\left(a_{u}^{(1)}, Y_{u} \mid a_{v}^{(2)}, \varnothing\right)=
$$




$$
\begin{aligned}
= & \prod_{u=2}^{n} \prod_{i=1}^{u-1}\left(\epsilon_{2}^{n-1}(-)^{i} i !(n-1-i) !\right)^{Y_{u, u-i}} \times \\
& \times \prod_{u=2}^{n} \prod_{i=0}^{u-2} \prod_{j=1}^{Y_{u, i+1}} \epsilon_{1}\left(\eta_{u-i}^{(1)}-\eta_{u}+\frac{\eta^{(2)}}{n}+j-i-1\right) .
\end{aligned}
$$

The derivation of the denominator of (2.2) though somewhat lengthier but still is quite straightforward and leads to

$$
\begin{aligned}
& \prod_{u=1}^{n} \prod_{v=1}^{n} Z_{b f}\left(a_{u}^{(1)} Y_{u} \mid a_{v}^{(1)} Y_{v}\right)= \\
& =\prod_{l=1}^{n-1} \prod_{v=1}^{n-l}\left(-\epsilon_{1}\right)^{Y_{v+l, l}} \prod_{k=l}^{v+l-1} \prod_{i=1+Y_{v+l, k+1}}^{Y_{v+l, k}}\left(\eta_{v+l}-\eta_{v}+l+Y_{v, k-l+1}-i\right) \times \\
& \times \prod_{l=0}^{n-2} \prod_{v=l+2}^{n} \epsilon_{1}^{Y_{v, l+1}} \prod_{k=l+1}^{v-1} \prod_{i=1+Y_{v, k+1}}^{Y_{v, k}}\left(\eta_{v}-\eta_{v-l}+l+1+Y_{v-l, k-l}-i\right) \times \\
& \times \prod_{v=2}^{n} \prod_{i=1}^{v-1}\left(\epsilon_{2}^{n-1}(-)^{i-1}(i-1) !(n-i) !\right)^{Y_{v, v-i}}\left((-)^{n-v-1-i}(n-v-1-i) !(v-i) ! \epsilon_{2}^{(n-1)}\right)^{Y_{v, i}},
\end{aligned}
$$

where the products on the second (third) line comes from the terms $u<v(u>v)$ and the last line results in from diagonal $u=v$ terms. Notice that, as we have already proved earlier, the order in $\epsilon_{1}$ of the numerator and the denominator coincide safely providing a finite $\epsilon_{1} \rightarrow$ 0 limit. Also dependence of the ratio in $\epsilon_{2}$ disappears (as it should from scaling arguments). Inserting (2.31), (2.32) and (2.33) in (2.2) for $F_{\vec{Y}}$ in the light asymptotic limit we finally get

$$
\begin{aligned}
& F_{\vec{Y}}=\prod_{u=2}^{n} \prod_{v=2}^{u}\left(\frac{u-v+1}{n-u+v-1}\right)^{Y_{u, v-1}} \\
& \times \frac{\prod_{i=0}^{Y_{u, u-v+1}-1}\left(-\eta_{u}+\eta_{v}^{(4)}+\frac{\eta^{(3)}}{n}-u+v+i\right)\left(\eta_{u}-\eta_{v}^{(1)}-\frac{\eta^{(2)}}{n}+u-v-i\right)}{\prod_{k=u-v+1}^{u-1} \prod_{i=Y_{u, k+1}}^{Y_{u, k}-1}\left(\eta_{u}-\eta_{v-1}+u-v+Y_{v-1, k+v-u}-i\right)\left(\eta_{u}-\eta_{v}+u-v+Y_{v, k+v-u}-i\right)} .
\end{aligned}
$$

where $Y_{v, i}$ is the number of boxes in the $i$ th row of diagram $Y_{v}$. As we have mentioned already, with the prescription (2.18) of the field, in the light asymptotic limit the prefactor in (2.15) becomes 1 , and hence, remembering also that the field $V_{\left(n q-b \eta^{(2)}\right) \omega_{1}}$ is equivalent to $V_{b \eta^{(2)} \omega_{n-1}}$, we can write

$$
{ }^{L} \mathcal{F}_{\eta}\left[\begin{array}{cc}
\eta^{(3)} \omega_{1} & \eta^{(2)} \omega_{n-1} \\
\eta^{(4)} & \eta^{(1)}
\end{array}\right](z) \equiv \lim _{\mathrm{b} \rightarrow 0} \mathcal{F}_{b \eta}\left[\begin{array}{cc}
b \eta^{(3)} \omega_{1} & b \eta^{(2)} \omega_{n-1} \\
b \eta^{(4)} & b \eta^{(1)}
\end{array}\right](z)=\sum_{\vec{Y}} F_{\vec{Y}} z^{|\vec{Y}|}
$$

The sum is taken over all Young diagrams $Y_{u}, u=2, \ldots, n$, with at most $u-1$ rows, i.e. over all allowed row lengths $Y_{u, 1} \geq Y_{u, 2} \geq \cdots \geq Y_{u, u-1} \geq 0$.

Let us consider the particular cases when $n=2$ (Liouville) and $n=3$ separately. 
When $n=2$ we have a single sum

$$
\begin{aligned}
L_{\mathcal{F}_{\eta}}^{\text {Liouv }}\left[\begin{array}{cc}
\eta^{(3)} & \eta^{(2)} \\
\eta^{(4)} & \eta^{(1)}
\end{array}\right](z) & =\sum_{i=0}^{\infty} \frac{\left(-\eta^{(4)}+\eta+\frac{\eta^{(3)}}{2}\right)_{i}\left(-\eta^{(1)}+\eta+\frac{\eta^{(2)}}{2}\right)_{i}}{i !(2 \eta)_{i}} z^{i} \\
& ={ }_{2} F_{1}\left(-\eta^{(1)}+\eta+\frac{\eta^{(2)}}{2},-\eta^{(4)}+\eta+\frac{\eta^{(3)}}{2}, 2 \eta ; z\right)
\end{aligned}
$$

where ${ }_{2} F_{1}(a, b ; c ; x)$ is the Gauss hyper-geometric function, and we set in $(2.36) \eta^{(4)} \equiv \eta_{1}^{(4)}$, $\eta^{(1)} \equiv \eta_{1}^{(1)}$ and $\eta \equiv \eta_{1}$. This is a well known result in Liouville theory [25-28].

When $n=3$ we get

$$
\begin{aligned}
& { }_{\mathcal{F}_{\eta} W_{3}}\left[\begin{array}{cc}
\eta^{(3)} \omega_{1} & \eta^{(2)} \omega_{2} \\
\eta^{(4)} & \eta^{(1)}
\end{array}\right](z)=\sum_{i, j, l=0}^{\infty}(-)^{l} 2^{j-i} z^{2 l+i+j} \\
& \times\left(\frac{\eta^{(3)}}{3}-\eta_{2}+\eta_{2}^{(4)}\right)_{i}\left(\frac{\eta^{(3)}}{3}-\eta_{3}+\eta_{2}^{(4)}-1\right)_{l}\left(\frac{\eta^{(3)}}{3}-\eta_{3}+\eta_{3}^{(4)}\right)_{j+l} \\
& \times \frac{\left(\frac{\eta^{(2)}}{3}-\eta_{2}+\eta_{2}^{(1)}\right)_{i}\left(\frac{\eta^{(2)}}{3}-\eta_{3}+\eta_{2}^{(1)}-1\right)_{l}\left(\frac{\eta^{(2)}}{3}-\eta_{3}+\eta_{3}^{(1)}\right)_{j+l}}{i ! j ! l !\left(\eta_{1}-\eta_{2}\right)_{i}\left(\eta_{1}-\eta_{3}-1\right)_{l}\left(\eta_{2}-\eta_{3}\right)_{l}\left(\eta_{2}-\eta_{3}-i-1\right)_{l}\left(\eta_{2}-\eta_{3}+l-i\right)_{j}}
\end{aligned}
$$

This formula completes the result of [26] where the light four-point function of $W_{3}$-theory has been computed in the case when both the second and the third insertions were light primaries of the same sort:

$$
\lambda^{(3)}=b \eta^{(3)} ; \quad \lambda^{(2)}=b \eta^{(2)}
$$

whereas (2.37) is obtained with the choice specified in (2.18). In the next section we present an alternative calculation of (2.37) based on the integral representation of the conformal blocks in the light asymptotic limit used in [26].

\section{Light asymptotic limit for the four point block in $W_{3}$}

It has been shown in [26] that the multi-point conformal blocks of the $W_{3}$ theory in the light asymptotic limit can be constructed in the terms of $s l(3)$ three-point invariant functions. For the details we refer the reader to the original paper. Here we'll introduce the necessary notations and state the relevant results.

It is well known that the $\operatorname{sl}(3)$ generators can be represented as operators acting on the triple of the isospin variables $Z=(w, x, y)$. To construct a multi-point block one should multiply several three-point functions then identify pairs of isospin variables corresponding to the internal states and integrate them out with an appropriate measure. At the end one specializes the external leg variables putting

$$
Z=\left(\frac{1}{2} z^{2}, z, z\right)
$$


where $z$ is the insertion point.

In particular the light asymptotic limit of the four-point block of the same type as in (2.37) can be represented as:

$$
\mathcal{F}=\int_{C} d^{3} Z_{s} \mathcal{E}_{1}\left(j_{2}, j_{1}, J_{s}^{\omega} \mid Z_{2}, Z_{1}, Z_{s}\right) \mathcal{E}_{2}\left(j_{3}, j_{4}^{\omega}, J_{s}^{* \omega} \mid Z_{3}, Z_{4}, Z_{s}\right),
$$

where $\mathcal{E}_{1}$ and $\mathcal{E}_{2}$ are the appropriate three point invariants given by ${ }^{1}$

$$
\begin{aligned}
& \mathcal{E}_{1}\left(j_{1}, j_{2}, j_{3} \mid Z_{1}, Z_{2}, Z_{3}\right)=\chi_{123}^{-J} \rho_{12}^{-J-r_{2}+s_{3}} \rho_{13}^{-J-r_{3}+s_{2}} \rho_{23}^{J-s_{2}} \rho_{32}^{J-s_{3}} \\
& \mathcal{E}_{2}\left(j_{1}, j_{2}, j_{3} \mid Z_{1}, Z_{2}, Z_{3}\right)=\sigma_{123}^{J} \rho_{21}^{J+r_{3}-s_{2}} \rho_{31}^{J+r_{2}-s_{3}} \rho_{23}^{-J-r_{3}} \rho_{32}^{-J-r_{2}}
\end{aligned}
$$

with

$$
\begin{aligned}
\rho_{i j}= & y_{i}\left(x_{i}-x_{j}\right)-\left(w_{i}-w_{j}\right) \\
\sigma_{i j k}= & x_{i} w_{j}-w_{i} x_{j}-x_{i} w_{k}+w_{i} x_{k}-w_{j} x_{k}+x_{j} w_{k} ; \\
\chi_{i j k}= & y_{i} w_{j}-w_{i} y_{j}+y_{i} y_{j}\left(x_{i}-x_{j}\right)-y_{i} w_{k}+w_{i} y_{k}+y_{i} y_{k}\left(x_{k}-x_{i}\right) \\
& -w_{j} y_{k}+y_{j} w_{k}+y_{j} y_{k}\left(x_{j}-x_{k}\right),
\end{aligned}
$$

the quantities $j=(r, s), j^{*}=(2-r, 2-s), j^{\omega}=(s, r)$ (see [26]) specify the primary fields and are related to the charge vectors $\eta_{u}$ introduced in section (2.3) as

$$
r=\eta_{1}-\eta_{2} ; \quad s=\eta_{2}-\eta_{3} ; \quad \eta_{1}+\eta_{2}+\eta_{3}=0
$$

and, finally,

$$
J=\left(h_{2}, j_{1}+j_{2}+j_{3}\right)=\frac{1}{3}\left(s_{1}+s_{2}+s_{3}-r_{1}-r_{2}-r_{3}\right) .
$$

Due to (3.5) and (2.16), (2.18) for our case we have

$$
\begin{array}{ll}
r_{s}=\eta_{1}-\eta_{2} ; & s_{s}=\eta_{2}-\eta_{3} ; \\
r_{1}=\eta_{1}^{(1)}-\eta_{2}^{(1)} ; & s_{1}=\eta_{2}^{(1)}-\eta_{3}^{(1)} ; \\
r_{4}=\eta_{1}^{(4)}-\eta_{2}^{(4)} ; & s_{4}=\eta_{2}^{(4)}-\eta_{3}^{(4)} ; \\
s_{2}=\eta^{(2)} ; & r_{2}=0 ; \\
r_{3}=\eta^{(3)} ; & s_{3}=0 .
\end{array}
$$

As usual, using projective invariance we can specify the insertion points as $\left(z_{4}, z_{3}, z_{2}, z_{1}\right) \rightarrow(\infty, 1, x, 0)$, see figure 2 . Under this specification, after dropping out an unimportant constant (infinite ) factor, $\mathcal{E}_{2}$ gets simplified

$$
\mathcal{E}_{2}\left(j_{3}, j_{4}^{\omega}, J_{s}^{* \omega} \mid Z_{3}, Z_{4}, Z_{s}\right)=\left(1-x_{s}\right)^{\frac{1}{3}\left(r_{4}+s_{s}-r_{s}-r_{3}-s_{4}\right)} \rho_{s, 3}^{\frac{r_{4}+s_{s}-r_{s}-r_{3}-s_{4}}{3}+s_{4}+r_{s}-2} .
$$

Putting

$$
\begin{aligned}
& x_{2} \rightarrow z ; \quad y_{2} \rightarrow z ; \quad w_{2} \rightarrow \frac{z^{2}}{2} ; \quad x_{1} \rightarrow 0 ; \\
& y_{1} \rightarrow 0 ; \quad w_{1} \rightarrow 0 ; \quad x_{3} \rightarrow 1 ; \quad y_{3} \rightarrow 1 ; w_{3} \quad \rightarrow \frac{1}{2},
\end{aligned}
$$

\footnotetext{
${ }^{1}$ We have different three point invariants, since the second and third light fields are of different kinds as specified in (2.18). The case of fields of the same kind is analysed in [26].
} 
as instructed in (3.1) and dropping out the usual factor $z^{h_{\alpha_{s}}-h_{\alpha^{(1)}}-h_{\lambda}(2) \omega_{1}}=$ $z^{r_{s}+s_{s}-\left(r_{1}+s_{1}\right)-s_{2}}$, up to an unimportant constant multiplier we get the integral

$$
\begin{aligned}
& \mathcal{F}=\int d x_{s} d y_{s} d w_{s}\left(w_{s}-y_{s}\left(x_{s}-\frac{z}{2}\right)\right)^{\frac{1}{3}\left(r_{1}+s_{s}-s_{1}-s_{2}-r_{s}\right)} \\
& \times w_{s}^{\frac{1}{3}\left(-r_{1}-s_{s}-2 s_{1}+s_{2}+r_{s}\right)}\left(1-x_{s}\right)^{\frac{1}{3}\left(-r_{3}-s_{4}+s_{s}+r_{4}-r_{s}\right)}\left(w_{s}-x_{s} y_{s}\right)^{\frac{1}{3}\left(-r_{1}-s_{s}+s_{1}+s_{2}-2 r_{s}\right)} \\
& \times\left(w_{s}-z\left(x_{s}-\frac{z}{2}\right)\right)^{\frac{1}{3}\left(r_{1}-2 s_{s}+2 s_{1}-s_{2}-r_{s}\right)}\left(w_{s}-\left(x_{s}-1\right) y_{s}-\frac{1}{2}\right)^{\frac{1}{3}\left(-r_{3}+2 s_{4}+s_{s}+r_{4}+2 r_{s}\right)-2} .
\end{aligned}
$$

After the change of the variables

$$
x_{s} \rightarrow \frac{x}{2 w} ; \quad w_{s} \rightarrow \frac{1}{2 w} ; \quad y_{s} \rightarrow \frac{y}{x y-w}
$$

we'll get

$$
\begin{aligned}
\mathcal{F}= & \int_{\mathcal{C}} d x d y d w w^{\frac{1}{3}\left(r_{3}+s_{4}+2 s_{s}-r_{4}+r_{s}\right)-2}(1-y z)^{\frac{1}{3}\left(r_{1}+s_{s}-s_{1}-s_{2}-r_{s}\right)} \\
& \times\left(w-\frac{x}{2}\right)^{\frac{1}{3}\left(-r_{3}-s_{4}+s_{s}+r_{4}-r_{s}\right)}\left(w z^{2}-x z+1\right)^{\frac{1}{3}\left(r_{1}-2 s_{s}+2 s_{1}-s_{2}-r_{s}\right)} \\
& \times(x y-w)^{\frac{1}{3}\left(r_{3}-2 s_{4}-s_{s}-r_{4}+r_{s}\right)}(-w+(x-2) y+1)^{\frac{1}{3}\left(-r_{3}+2 s_{4}+s_{s}+r_{4}+2 r_{s}\right)-2} .
\end{aligned}
$$

Here is the result of the integration (for the details of the calculation see appendix: A)

$$
\begin{aligned}
& \mathcal{F}=\sum_{m, n, k=0}^{\infty} \sum_{l=0}^{m}(-)^{k+l} 2^{n-m} z^{2 k+m+n} \\
& \times\left(\frac{1}{3}\left(s_{s}+2 r_{s}-r_{3}-s_{4}+r_{4}\right)\right)_{l}\left(\frac{1}{3}\left(-s_{s}+r_{s}-r_{1}+s_{1}+s_{2}\right)\right)_{m} \\
& \times \frac{\left(\frac{1}{3}\left(2 s_{s}+r_{s}-r_{1}-2 s_{1}+s_{2}\right)\right)_{k+n}\left(\frac{1}{3}\left(2 s_{s}+r_{s}+r_{3}-2 s_{4}-r_{4}\right)\right)_{k+n}\left(\frac{1}{3}\left(2 s_{s}+r_{s}+r_{3}+s_{4}-r_{4}-3\right)\right)_{k-l+m}}{k ! ! ! n !(m-l) !\left(r_{s}\right)_{l}\left(s_{s}\right)_{k-l+n}\left(s_{s}+r_{s}-1\right)_{k+m}} .
\end{aligned}
$$

Though this expression looks different from (2.37) below we argue that in fact they coincide. First we will prove this analytically up to the second order in $z$. It is convenient to rewrite (3.12) and (2.37) in terms of parameters $A_{1}, A_{2}, B_{1}, B_{2}$ defined as

$$
\begin{array}{rlrl}
A_{1} & =\frac{1}{3}\left(r_{s}-r_{1}-s_{s}+s_{1}+s_{2}\right) ; & B_{1}=\frac{1}{3}\left(r_{s}-r_{1}+2 s_{s}-2 s_{1}+s_{2}\right) ; \\
A_{2}=\frac{1}{3}\left(r_{s}+r_{3}+s_{4}-s_{s}-r_{4}\right) ; & B_{2}=\frac{1}{3}\left(r_{s}+r_{3}-2 s_{4}+2 s_{s}-r_{4}\right) .
\end{array}
$$

For (3.12) we will get

$$
\mathcal{F}=\sum_{k, n, m=0}^{\infty} \sum_{l=0}^{m} \frac{(-1)^{k+l} 2^{n-m}\left(A_{1}\right)_{m}\left(r_{s}-A_{2}\right)_{l}\left(B_{1}\right)_{k+n}\left(B_{2}\right)_{k+n}\left(A_{2}+s_{s}-1\right)_{k-l+m} z^{2 k+m+n}}{k ! l ! n !(m-l) !\left(r_{s}\right)_{l}\left(s_{s}\right)_{k-l+n}\left(r_{s}+s_{s}-1\right)_{k+m}}
$$

and (2.37) is given

$$
{ }^{L} \mathcal{F}_{W_{3}}=\sum_{k, n, m=0}^{\infty} \frac{(-1)^{k} 2^{m-n}\left(A_{1}\right)_{n}\left(A_{2}\right)_{n}\left(A_{1}+s_{s}-1\right)_{k}\left(A_{2}+s_{s}-1\right)_{k}\left(B_{1}\right)_{k+m}\left(B_{2}\right)_{k+m} z^{2 k+m+n}}{k ! m ! n !\left(s_{s}\right)_{k}\left(r_{s}\right)_{n}\left(-n+s_{s}-1\right)_{k}\left(r_{s}+s_{s}-1\right)_{k}\left(k-n+s_{s}\right)_{m}}
$$


It is easy to see from (3.15) that the term proportional to $z$ is

$$
\mathcal{F}^{(1)}=\frac{A_{1}\left(A_{2}+s_{s}-1\right)}{2\left(r_{s}+s_{s}-1\right)}-\frac{A_{1}\left(s_{s}-1\right)\left(r_{s}-A_{2}\right)}{2 r_{s}\left(r_{s}+s_{s}-1\right)}+\frac{2 B_{1} B_{2}}{s_{s}}
$$

and for (3.16) it is

$$
{ }^{L} \mathcal{F}_{W_{3}}^{(1)}=\frac{A_{1} A_{2}}{2 r_{s}}+\frac{2 B_{1} B_{2}}{s_{s}}
$$

Combining the first two terms in (3.17) we will get (3.18). The details of the second order calculations can be found in appendix B.

Using Mathematica code we have checked up to the 8th order in $z$, that (2.37) agrees with (3.12).

Note that the expression (2.37), besides the physical poles at $r_{s} \in \mathbb{Z}_{\leq 0}, s_{s} \in \mathbb{Z}_{\leq 0}$, $r_{s}+s_{s}-1 \in \mathbb{Z}_{\leq 0}$, has apparent poles at the positive integer values of $s_{s}$. In fact explicit calculations ensure that these apparent poles get cancelled in the final expressions.

\section{Discussion}

The methods developed in this paper can be extended to other cousins of the Liouville and Toda field theories, like super Liouville and super Toda. Also it is an important problem to compute the conformal blocks in the light asymptotic limit for an arbitrary $n$ via the integral representation, as it was done for $A_{2}$ Toda field theory in the last section.

Let us also recall that in the light asymptotic limit the $W_{n}$ symmetry reduces to $\operatorname{SL}(n)$ group. This means that the function (2.34) in fact should have a group theoretical meaning. It would be interesting to discover it.

\section{Acknowledgments}

The work of R.P. and G.S. was partially supported by the Armenian SCS grant 15T-1C308 and by ICTP OEA-AC-100 project. The work of H.P. was partially supported by the Armenian SCS grant 15T-1C058. The work of G.S. was also supported by ICTP Network NET68. The work of H.P. and G.S. was partially supported by the ANSEF grant hepth-4208.

\section{A The integral calculation}

For a suitable integration contour it is allowed to have boundaries ending on branch points of the integrand of (3.11). Another necessary condition is that the result of integration should be analytic in insertion point $z$. Here is an appropriate choice for contour $\mathcal{C}$, satisfying both requirements

$$
\mathcal{C}: \quad y \in\left(\frac{w}{x}, \frac{w-1}{x-2}\right) \text { then } w \in\left(0, \frac{x}{2}\right) \text { then } x \in(0,2)
$$

There are to factors in integrand of (3.11) which depend on $z$. Expanding the product of these factors in powers of $z$ we get

$$
(1-y z)^{g}\left(1-x z+w z^{2}\right)^{h}=\sum_{m, k, n=0}^{\infty} C_{m, k, n} z^{2 k+m+n} x^{n} w^{k} y^{m},
$$


where

$$
C_{m, k, n}=(-)^{m+n} \frac{\Gamma(g+1) \Gamma(h+1)}{m ! k ! n ! \Gamma(g-m+1) \Gamma(h-k-n+1)} .
$$

Inserting this into (3.11) we'll find

$$
\begin{aligned}
\mathcal{F}= & \sum_{m, k, n=0}^{\infty} C_{m, k, n} z^{2 k+m+n} \int d x d y d w \\
& \times y^{m} x^{n} w^{e+k}\left(w-\frac{x}{2}\right)^{f}(x y-w)^{A}(-w+(x-2) y+1)^{B},
\end{aligned}
$$

where we introduced the notations

$$
\begin{aligned}
e & =\frac{1}{3}\left(r_{3}+s_{4}+2 s_{s}-r_{4}+r_{s}\right)-2 ; & f & =\frac{1}{3}\left(-r_{3}-s_{4}+s_{s}+r_{4}-r_{s}\right) ; \\
A & =\frac{1}{3}\left(r_{3}-2 s_{4}-s_{s}-r_{4}+r_{s}\right) ; & B & =\frac{1}{3}\left(-r_{3}+2 s_{4}+s_{s}+r_{4}+2 r_{s}\right)-2 ; \\
g & =\frac{1}{3}\left(r_{1}+s_{s}-s_{1}-s_{2}-r_{s}\right) ; & h & =\frac{1}{3}\left(r_{1}-2 s_{s}+2 s_{1}-s_{2}-r_{s}\right) .
\end{aligned}
$$

Inserting binomial expansion

$$
y^{m}=\sum_{l=0}^{m}\left(\frac{w}{x}\right)^{m-l}\left(y-\frac{w}{x}\right)^{l}\left(\begin{array}{c}
m \\
l
\end{array}\right)
$$

and shifting the variable $y \rightarrow y+\frac{w}{x}$ we'll get the integral

$$
\begin{aligned}
\mathcal{F} & =\sum_{m, k, n=0}^{\infty} C_{m, k, n} z^{2 k+m+n} \int d x d y d w \\
& =\sum_{l=0}^{m} x^{A}(x-2)^{B} x^{n} y^{A+l} w^{e+k}\left(w-\frac{x}{2}\right)^{f}\left(y-\frac{2 w-x}{x(x-2)}\right)^{B}\left(\frac{w}{x}\right)^{m-l}\left(\begin{array}{c}
m \\
l
\end{array}\right) .
\end{aligned}
$$

The result of integration over $y \in\left[0, \frac{2 w-x}{x(x-2)}\right]$ is

$$
\begin{aligned}
\mathcal{F}= & \sum_{m, k, n=0}^{\infty} C_{m, k, n} z^{2 k+m+n} \sum_{l=0}^{m} 2^{l}\left(\begin{array}{c}
m \\
l
\end{array}\right) \frac{\Gamma(B+1) \Gamma(A+l+1)}{\Gamma(A+B+l+2)} \\
& \times \int d x d w(x-2)^{-A-l-1} x^{-B-m+n-1}\left(w-\frac{x}{2}\right)^{A+B+f+l+1} w^{e+k-l+m} .
\end{aligned}
$$

Next we'll integrate over $w \in[0, x / 2]$ and get

$$
\begin{aligned}
& \mathcal{F}=\sum_{m, k, n=0}^{\infty} C_{m, k, n} 2^{n-m} z^{2 k+m+n} \sum_{l=0}^{m}\left(\begin{array}{c}
m \\
l
\end{array}\right) \frac{\Gamma(B+1) \Gamma(A+l+1)}{\Gamma(A+B+l+2)} \\
& \times \frac{\Gamma(A+B+f+l+2) \Gamma(e+k-l+m+1)}{\Gamma(A+B+e+f+k+m+3)} \int d x\left(1-\frac{x}{2}\right)^{-A-l-1}\left(\frac{x}{2}\right)^{A+e+f+k+n+1} .
\end{aligned}
$$

The last integral over $x \in[0,2]$ is again of Euler type so that the final result is

$$
\mathcal{F}=\sum_{m, k, n=0}^{\infty} C_{m, k, n} 2^{n-m} z^{2 k+m+n} \sum_{l=0}^{m}\left(\begin{array}{c}
m \\
l
\end{array}\right) \frac{\Gamma(B+1) \Gamma(A+l+1)}{\Gamma(A+B+l+2)}
$$




$$
\times \frac{\Gamma(A+B+f+l+2) \Gamma(e+k-l+m+1)}{\Gamma(A+B+e+f+k+m+3)} \frac{\Gamma(-A-l) \Gamma(A+e+f+k+n+2)}{\Gamma(e+f+k-l+n+2)} .
$$

It remains to use (A.5) to arrive at (3.12).

\section{B The second order terms for $W_{3}$}

From (3.15) the term proportional to $z^{2}$ is

$$
\begin{aligned}
\mathcal{F}^{(2)}= & -\frac{B_{1} B_{2}\left(A_{2}+s_{s}-1\right)}{s_{s}\left(r_{s}+s_{s}-1\right)}+\frac{2 B_{1}\left(B_{1}+1\right) B_{2}\left(B_{2}+1\right)}{s_{s}\left(s_{s}+1\right)}+ \\
& \left.+\frac{A_{1} B_{1} B_{2}\left(A_{2}+s_{s}-1\right)}{s_{s}\left(r_{s}+s_{s}-1\right)}-\frac{A_{1} B_{1} B_{2}\left(r_{s}-A_{2}\right)}{r_{s}\left(r_{s}+s_{s}-1\right)}+\frac{A_{1}\left(A_{1}+1\right)\left(A_{2}+s_{s}-1\right)\left(A_{2}+s_{s}\right)}{8\left(r_{s}+s_{s}-1\right)\left(r_{s}+s_{s}\right)} \quad \text { (B. } 1\right) \\
& -\frac{A_{1}\left(A_{1}+1\right)\left(s_{s}-1\right)\left(r_{s}-A_{2}\right)\left(A_{2}+s_{s}-1\right)}{4 r_{s}\left(r_{s}+s_{s}-1\right)\left(r_{s}+s_{s}\right)}+\frac{A_{1}\left(A_{1}+1\right)\left(s_{s}-2\right)\left(s_{s}-1\right)\left(r_{s}-A_{2}\right)\left(-A_{2}+r_{s}+1\right)}{8 r_{s}\left(r_{s}+1\right)\left(r_{s}+s_{s}-1\right)\left(r_{s}+s_{s}\right)}
\end{aligned}
$$

and for (3.16) it is

$$
\begin{aligned}
{ }^{L} \mathcal{F}_{W_{3}}^{(2)}= & -\frac{B_{1} B_{2}\left(A_{1}+s_{s}-1\right)\left(A_{2}+s_{s}-1\right)}{\left(s_{s}-1\right) s_{s}\left(r_{s}+s_{s}-1\right)}+\frac{A_{1}\left(A_{1}+1\right) A_{2}\left(A_{2}+1\right)}{8 r_{s}\left(r_{s}+1\right)}+ \\
& +\frac{A_{1} A_{2} B_{1} B_{2}}{r_{s}\left(s_{s}-1\right)}+\frac{2 B_{1}\left(B_{1}+1\right) B_{2}\left(B_{2}+1\right)}{s_{s}\left(s_{s}+1\right)}
\end{aligned}
$$

Combining the last three terms in (B.1) we will get the second term in (B.2), the second term of (B.1) coincides with the last term in (B.2), and finally the sum of the first, third and the forth terms in (B.1) coincides with the sum of the first and the third terms in (B.2).

Note that the first and third terms in the expression (B.2)) suffer from the apparent spurious poles at $s_{s}-1=0$, which get cancelled when combined. Similarly the last three terms in (B.1) have apparent but at this time physical pole at $r_{s}+s_{s}=0$, which as well get cancelled when combined. It is curious that together with the expected cancellation of the apparent spurious pole, we have sometimes also cancellation of the physical poles.

Open Access. This article is distributed under the terms of the Creative Commons Attribution License (CC-BY 4.0), which permits any use, distribution and reproduction in any medium, provided the original author(s) and source are credited.

\section{References}

[1] E. Braaten, T. Curtright, G. Ghandour and C.B. Thorn, Nonperturbative weak coupling analysis of the quantum Liouville field theory, Annals Phys. 153 (1984) 147 [INSPIRE].

[2] E. Braaten, T. Curtright and C.B. Thorn, An exact operator solution of the quantum Liouville field theory, Annals Phys. 147 (1983) 365 [INSPIRE].

[3] C.B. Thorn, Liouville perturbation theory, Phys. Rev. D 66 (2002) 027702 [hep-th/0204142] [INSPIRE].

[4] V.A. Fateev and A.V. Litvinov, Correlation functions in conformal Toda field theory. I, JHEP 11 (2007) 002 [arXiv: 0709.3806] [INSPIRE]. 
[5] N. Seiberg, Notes on quantum Liouville theory and quantum gravity, Prog. Theor. Phys. Suppl. 102 (1990) 319 [INSPIRE].

[6] A.B. Zamolodchikov and A.B. Zamolodchikov, Structure constants and conformal bootstrap in Liouville field theory, Nucl. Phys. B 477 (1996) 577 [hep-th/9506136] [INSPIRE].

[7] V. Fateev and S. Ribault, Conformal Toda theory with a boundary, JHEP 12 (2010) 089 [arXiv: 1007.1293] [INSPIRE].

[8] P. Menotti and E. Tonni, Liouville field theory with heavy charges. I. The pseudosphere, JHEP 06 (2006) 020 [hep-th/0602206] [INSPIRE].

[9] P. Menotti and E. Tonni, Liouville field theory with heavy charges. II. The conformal boundary case, JHEP 06 (2006) 022 [hep-th/0602221] [INSPIRE].

[10] D. Harlow, J. Maltz and E. Witten, Analytic continuation of Liouville theory, JHEP 12 (2011) 071 [arXiv:1108.4417] [INSPIRE].

[11] H. Poghosyan and G. Sarkissian, On classical and semiclassical properties of the Liouville theory with defects, JHEP 11 (2015) 005 [arXiv: 1505.00366] [INSPIRE].

[12] L.F. Alday, D. Gaiotto and Y. Tachikawa, Liouville correlation functions from four-dimensional gauge theories, Lett. Math. Phys. 91 (2010) 167 [arXiv:0906.3219] [INSPIRE].

[13] N. Wyllard, $A_{N-1}$ conformal Toda field theory correlation functions from conformal $N=2$ $\mathrm{SU}(N)$ quiver gauge theories, JHEP 11 (2009) 002 [arXiv:0907.2189] [INSPIRE].

[14] V.A. Alba, V.A. Fateev, A.V. Litvinov and G.M. Tarnopolskiy, On combinatorial expansion of the conformal blocks arising from AGT conjecture, Lett. Math. Phys. 98 (2011) 33 [arXiv: 1012.1312] [INSPIRE].

[15] V.A. Fateev and A.V. Litvinov, Integrable structure, $W$-symmetry and AGT relation, JHEP 01 (2012) 051 [arXiv:1109.4042] [INSPIRE].

[16] A. Lossev, N. Nekrasov and S.L. Shatashvili, Testing Seiberg-Witten solution, in Strings, branes and dualities. Proceedings, NATO Advanced Study Institute, Cargese France May 26-June 141997 [hep-th/9801061] [INSPIRE].

[17] N.A. Nekrasov, Seiberg-Witten prepotential from instanton counting, Adv. Theor. Math. Phys. 7 (2003) 831 [hep-th/0206161] [INSPIRE].

[18] L.F. Alday, D. Gaiotto, S. Gukov, Y. Tachikawa and H. Verlinde, Loop and surface operators in $N=2$ gauge theory and Liouville modular geometry, JHEP 01 (2010) 113 [arXiv: 0909.0945] [INSPIRE].

[19] R. Poghossian, Recursion relations in CFT and $N=2$ SYM theory, JHEP 12 (2009) 038 [arXiv: 0909.3412] [INSPIRE].

[20] R. Flume and R. Poghossian, An algorithm for the microscopic evaluation of the coefficients of the Seiberg-Witten prepotential, Int. J. Mod. Phys. A 18 (2003) 2541 [hep-th/0208176] [INSPIRE].

[21] U. Bruzzo, F. Fucito, J.F. Morales and A. Tanzini, Multiinstanton calculus and equivariant cohomology, JHEP 05 (2003) 054 [hep-th/0211108] [INSPIRE].

[22] A. Marshakov, A. Mironov and A. Morozov, On AGT relations with surface operator insertion and stationary limit of beta-ensembles, J. Geom. Phys. 61 (2011) 1203 [arXiv: 1011.4491] [INSPIRE]. 
[23] M. Piatek, Classical torus conformal block, $N=2^{*}$ twisted superpotential and the accessory parameter of Lamé equation, JHEP 03 (2014) 124 [arXiv:1309.7672] [INSPIRE].

[24] R. Poghossian, Deformed $S W$ curve and the null vector decoupling equation in Toda field theory, JHEP 04 (2016) 070 [arXiv: 1601.05096] [INSPIRE].

[25] A. Mironov and A. Morozov, Proving AGT relations in the large-c limit, Phys. Lett. B 682 (2009) 118 [arXiv:0909.3531] [INSPIRE].

[26] V. Fateev and S. Ribault, The large central charge limit of conformal blocks, JHEP 02 (2012) 001 [arXiv:1109.6764] [INSPIRE].

[27] N. Hama and K. Hosomichi, AGT relation in the light asymptotic limit, JHEP 10 (2013) 152 [arXiv:1307.8174] [INSPIRE].

[28] A.B. Zamolodchikov, Conformal symmetry in two-dimensions: an explicit recurrence formula for the conformal partial wave amplitude, Commun. Math. Phys. 96 (1984) 419 [INSPIRE].

[29] P. Bowcock and G.M.T. Watts, On the classification of quantum $W$ algebras, Nucl. Phys. B 379 (1992) 63 [hep-th/9111062] [INSPIRE]. 